\title{
Analysis of Inverse Methods in Empirical Structural Mechanics
}

\author{
ling pi Youn \\ School of Mechanical Engineering, Shanghai Jiao Tong University International Student Center, Shanghai, China. \\ youngpi5255@hotmail.com
}

\begin{abstract}
ArticleInfo
Journal of Machine and Computing (http://anapub.co.ke/journals/jmc/jmc.html)

Doi : https://doi.org/10.53759/7669/jmc202101021

Received 30 March 2021; Revised form 30 June 2021; Accepted 18 August 2021; Available online 05 October 2021. (O2021 The Authors. Published by AnaPub Publications.

This is an open access article under the CC BY-NC-ND license. (http://creativecommons.org/licenses/by-nc-nd/4.0/)

Abstract - The application of inverse methods in empirical structural mechanics is the subject of this study. After a broad introduction to Inverse Problems (IPs), which includes a discussion of the many domains of application in general structural mechanics, the focus is limited to the critical area of material identification, with a special focus on the use of complete surveys. In this example, a more detailed explanation of the IPs to solve is provided, as well as the primary approaches to solving it. Lastly, there are several illustrations of exploratory uses of such techniques.
\end{abstract}

Keywords - Inverse Problems (IPs), Finite Element Model Updating (FEMU), Virtual Fields Method (VFM), Structural Mechanics.

\section{INTRODUCTION}

People are spending about $89 \%$ total of their active time in enclosed spaces such as structures, cars, and other structures [1]. For inhabitants, a relatively pleasant, healthy, and constructive confined setting is critical. Heating, Ventilation, And Air-Conditioning (HVAC) systems are often used to manage the atmospheric temperature, humidity levels, air velocity, and morphological and chemical ratios in enclosed areas. HVAC systems consume more than 40 percent of the energy generated in a structure. The operational power of HVAC systems in a four hundred-passenger airplane may reach 350 $\mathrm{kW}$, accounting for around $75 \%$ of total non-propulsive energy demand on deck. The confined surroundings formed on the basis of such enormous energy demand and related greenhouse gas emissions are not desirable. The most common criticism of office workers within facilities, as per statistics from the International Facility Management Association, is that "it was too humid and also too frigid at the same time."

Not only is the thermal climate bad, but investigations have shown that up to $20 \%$ of our buildings may be affected by sick building syndrome. Heating and air conditioning system faults are often blamed for this ailment. Volatile organic compounds (VOCs) from outgassing building materials, molds, and mild industrial chemicals used in industries are all potential sources of poor indoor air quality. Up to half of passengers on commercial airplanes have health issues, with $6 \%$ needing medical care. In an aircraft cabin, respiratory illnesses are very contagious. For example, twenty two people were infected with SARS on a trip from Hong Kong to Beijing in 2003, and the H1N1-A virus spread to seventy six nations on six continents in less than a month and a half in 2009. Indoor settings, particularly in high-density buildings and transportation vehicles, may be improved further. In traditional structural mechanics, experimenters focus on describing the mechanical behavior of materials and structures using a set of constants or curves, while theoreticians focus on creating theories and models that are compatible with the curves and constants discovered in [2].

We are living in a highly unique age in the history of structural mechanics, in which models and experiments can be directly compared, thanks to exponential increases in computing capacity and the constant development of new and more sophisticated experimental methods. This opens up new possibilities for more precisely predicting the mechanical behavior of complex systems and materials. The parameters of the theoreticians' models may be deduced directly from the experiments. This does; however, need the solution of an "Inverse Problems (IPs)."

The first part of this paper's goal is to provide an outline of the basic difficulties concerning Inverse Problems (IPs) in empirical structural mechanics. Because the topic is so vast, this part will include extremely basic information as well as a number of important references to help interested readers get started [3]. The remainder of this article will be devoted to one of the most important applications of IPs in experimental structural mechanics: identifying the variables of mechanical fundamental behavioral principles of substances, with a particular emphasis on the use of full-field data. This paper has been organized as follows: Section II presents an analysis of applications for Inverse Problems (IPs). Section III focuses on practical inverse problems types. Section IV presents a theoretical investigation of material constitutive dynamics. Section $\mathrm{V}$ concludes the research.

\section{APPLICATION}

We may make predictions using the theories of mechanics and dynamics of deformable bodies: given a full description of a system, we can anticipate the results of certain observations. The forward issue is a challenge that involves anticipating the outcome of measurements. 
In the Inverse Problems (IPs), the values of the system's parameters are inferred from the actual results of certain measurements. The primary kinds of IPs that occur in deformable structural mechanics are comparable to those that develop in other fields of physics involving continuous media and dispersed physical variables, such as acoustics, electrostatics, and electromagnetic. They are typically driven by a desire or need to fill a knowledge gap about the system's characteristics (a deformable solid body or structure). This need or requirement may be found in a variety of mechanical areas, with many industrial and medical applications:

There are applications in geotechnical engineers, for example, for detecting tunnel oil or other natural resources; seismology, for example, for simulating seismic waves; astronautical engineering and space exploration, for example, for upgrading system dynamics of superstructures; resource mechanics, for example, for defining characteristics of materials; medicine, for example, for identifying tumors; and industrial production, for identifying errors. The following is a typical formulation of a forward problem: If $\mathrm{p}$ denotes a collection of parameter estimation and $\mathrm{u}$ denotes a set of observables (data), then $g$ denotes a polynomial operator that provides the error-free estimates of $u$, for instance:

$$
u=g(p)
$$

The basic Partial Differential Equations (PDEs) controlling the mechanical characteristics of materials and complexes of objects are solved to find the operator "g." It's possible to write these equations as follows:

$$
0=M(u, p)
$$

The forward constraint formulas (equilibrium formulas, constitutive formulae, and compatibility formulae), which include the explanatory variables and variable - length, are represented by $\mathrm{M}$ in the formula above. In quasi-static modeling, for instance, $\mathrm{M}$ has the following generic form:

$$
K(p)(u-F)=M(u, p)
$$

$\mathrm{K}$ is the elasticity function in the preceding formula, the measurements " $\mathrm{u}$ " are local dispersion variables, and " $F$ " are local force variables. The IP is to find the operators that give the combination of multiple variables $p$ that match to the observed data set $\mathrm{u}$, that is, the set of parameter estimation $\mathrm{p}$ that agree both with measurable data set $\mathrm{u}$ and the operator $\mathrm{M}$ in Eq (2). A nonlinear optimization issue is solved to produce components of the uncertain variables by formulating an objective function that eliminates the discrepancies or misfit between both the observable variables and those anticipated by the $g$ operators via PDEs.

The IP is frequently considerably more difficult to alleviate than the forward issue since: the forward remedy is simply a sub-problem of the iterative algorithm; the IP is frequently ill-posed notwithstanding the forward situation's wellposedness (a well-posed issue in arithmetic is one for which some scientific theories support the existence and analytical solution);

All of these challenges cause numerical problems when minimizing the objective functions. Different techniques may be employed depending on the size of the issue to be solved and its degree of ill-posedness: evolutionary algorithms, Newton's technique, quasi-Newton techniques (such as L-BFGS), and steepest drop. Optimization problem has always been limited: one must minimize the discrepancies between measurable and anticipated data while keeping in mind that the anticipated ones must fulfill the PDEs that produce Eq (1). Optimization is considered to be PDE-constrained. Inequality limits may also be used to limit the ranges of random variables.

Many Inverse Problems (IPs) are specified using a least-squares PDE-constrained optimization problem that is optimized using a gradient-based method (Quasi-Newton/ Newton). As a result, by demanding stationary properties of a Lagrangian integral, the formulas that govern the solutions may be obtained [4]. All these optimization problem requirements, in particular, assume the shape of a system of nonlinear three-field system of integro-partial-differential functions in the parameter $u$, the partial derivatives variable, and the prediction models " $p$ ".

Such a challenge is bad throughout the sense that the remedy cannot be specific and must rely on the information indefinitely. Antithesis contractors that are inadequate and ranks completely lacking are produced by discretizing the invertible issue. For two main reasons: error terms and system flaws, the projected variables cannot be equal to the calculated data in particular. As a result, without the need for a thorough examination of prototype and data errors, it is nearly impossible to appropriately set inverted issues. The activator is indeed not consistently bijective, implying that the same results of $\mathrm{u}$ may be forecasted using two distinct choices of design variables, or if some variables of $u$ cannot be anticipated using any combination of multiple criteria.

Certain obstacles can be resolved in a variety of ways: One way to make things more normal is to penalize extremely unlikely answers. Especially if some are of good hypothesis about the concept available may unlikely alternatives be ruled out; the quantity and relevance that a substantial level can determine any procedure's overall effectiveness. Whenever the uncertain hyper-parameters describe the distribution pattern of a fundamental feature that is recognized to fluctuate slowly, regularization with the sophomore of the slope of such dispersion could be beneficial. Wavelike features of $\mathrm{p}$ are ruled out by this Based semi - supervised normalization. J. Gunning and M. Sams in [5] have demonstrated that the Based semi - supervised method can ease out prototype inconsistencies and encourage the use of Markov random normalization as an effective substitute.

Some other option is to provide a statistical alternative to the analytical solution. Every number of criteria in the universe of potential parameters $\mathrm{p}$ is given likelihood. Probability densities reflecting a probable set of assumptions and limitations can be used to calculate this possibility. Hence median, mean, and expectation - maximization remedies can all be determined using the calculated chances. J. Zhang in [6] expresses the outcome in terms of periods as well. 
Investigations in [7] can be fine-tuned as a sensible approach. Because of insufficient concentration and placement of supplies (or input sites) and detectors (or assessment stations), the awareness of the optimization problem to a particular estimated variable might be totally reduced. By researching and adjusting variable tolerances, it is possible to improve these volumes or / and regions. Adjusting the exploratory approach could also assist solve some issues that are largely uniform. This could, therefore, have the opposite impact since, when revolutionary new procedures improve the ability to reduce scientific variability or increase survey frequency; fresh theoretical approaches with a higher space of possible factors emerge, allowing for much more precise accounting of the data.

Because there is typically no closed-form solution providing a direct relationship between readings and variable length, the provision of detection algorithms allowing the derivation of constitutive parameters is a major problem in such approaches.

In the past few years, many ways have been presented, including model update strategies and the so-called Virtual Field Methods (VFM). The basic elements of these techniques will be presented in this part, and Section III will demonstrate their relevance through a discussion of some modern and practical cases of implementation. Today, static structural researchers working on reverse issues have two primary options:

- The first is innovative: it involves reducing the remedy risk of conventional fractional derivative that have existed for years (establishment of innovative experimental methods, factorial of experiments conducted, introduction of innovative studies, version adjustments, and so forth);

- The other is complex and statistical: it involves arithmetical issues resulting from the settlement of conventional nonlinear systems that have existed for decades (emergence of new experimental studies, factorial of laboratory investigation, formation of new research projects, concept additions, and so on).

$\bullet$

\section{PRACTICAL INVERSE PROBLEMS (IPS) TYPES}

IPs may be classified according to the kind of variable sought. IPs in the dynamics of deformed materials may be classified into seven categories using this set of criteria:

1) The restoration of geometrical buried objects such as fractures, cavities, and inclusions. A crack (or a group of cracks) must be identified out of a combination of over-determined pressure limit metrics in crack diagnosis issues.

Electromagnetism, nonlinear elastic, acoustical, and elastodynamics are some of the physical settings in which such issues may be articulated. They have significant uses in non-destructive testing and the detection of seismic faults, for example. For crack detection issues, a variety of methods have been suggested. Crack detection issues may be defined and addressed using reciprocity gap functionals in particular.

2) Mechanical oscillations, - for example ultrasound, Rayleigh, or Lambert waves, are used in non-destructive testing (NDT) of objects or materials. Engineering systems and cultural artefacts have both benefited from NDT with waves. Waves of various frequencies may be employed, determined by the size of the elements under examination and the pixel pitch required.

The goal is to determine the frequency bands robust and dependable dispersion characteristics, particularly those of longer wavelengths. This may be accomplished by analyzing phase discrepancies between pulses of various frequencies at two distinct measurement locations.

3) The recognition of distributed parameters (e.g., elasticity modulus, mass density, and wavenumber) has applications in the fields e.g. radiography of materials, where the goal is to describe the rigidity of tissues in identifying illnesses or tumours, or in geophysical surveys and geomechanics, where the goal is to reconstitute the distributed statutory instruments of various geological formations.

K. You-Ten, D. Wong, X. Ye, C. Arzola, A. Zand and N. Siddiqui in [8] tried to identify hard tissues by external palpation a few years ago. Even though methods that combine external loadings and external measurements have proven effective in a variety of applications, it is now feasible to combine external loading with internal (bulk) tridimensional fullfield observations. It is feasible to detect displacement fields in various human tissues using ultrasonography and magnetic resonance imaging (MRI) in medical research. Experimental methods such as X-ray or electron diffraction radiography, or spectrum scan diffraction gratings have become accessible for bulk observations in material requirements in the area of materials dynamics.

4) In order to achieve the ultimate goal of constitutive estimating the parameters, powerful and efficient methods for analyzing this kind of full-field data must be devised. In medical disciplines, where full-field data have been accessible for more than a decade, a lot of progress has already been made. Elastography, or the measuring of tissue elasticity, has even become its own branch.

The methods for processing full-field data vary depending on how the solid is deformed: static experiments in which the tissue is condensed quasi-statically, and vibrant experimentations in which a period harmonic activation on the boundary produces a time harmonic shear wave in the tissue; The methods for processing full-field data vary depending on how the solid is disfigured: static tests in which the tissues are squeezed quasi-statically, and dynamic operations in which a time harmonic stimulation on the barrier causes a period rhythmic shear wave inside the tissues;

5) Modelling of cyclic loading, which has significant engineering consequences. Internal stresses may be measured using a variety of methods, including sensor devices such as optical fibers or strain gages.

The hole-drilling technique, layer removal method, and compliance approach may all be utilized on laminated synthetic structures at a ply or macroscopic scale. The compliance technique for filament wound tubes entails sectioning the tubes and using two biaxial strain gages, one attached to each end of the tube wall, to measure the change in stresses on the exterior and interior surfaces. This allows the radial and peripheral internal bend stresses of filaments woven cylinders 
to be calculated. Hole-drilling or other removal methods combined with complete dispersion field imaging technologies may provide several benefits over conventional strain gauge usage, including heightened sensitivity and no-contact assessment.

6) In the models of complicated engineering structures, the adjustment of local characteristics that are not recognized with adequate precision.

7) It is also necessary to identify sources of unreachable control limits (i.e., Cauchy issues in elasticity).

8) Eventually, the discovery of constitutive characteristics in solids and systems that defy simple assumptions like continuous levels of strain or stress. The literature on the six previous points is detailed, and there are many papers in the literature that cover the topic.

Due to advancement of full-field observations, the determination of intrinsic characteristics in solids and systems (stationary or slowly changing properties) has improved dramatically during the past decade. Such experimental methods provide a variety of material and are thus ideally suited to solving identification issues. As inversion methods that explicitly utilize the existence of domain dimensions (either on the perimeter or across a portion of the domains) are available, the significance of the latter approaches is growing. Because of the significance of these inversion methods, as well as their advanced technologies, the remainder of the article will be devoted entirely to them.

\section{THEORETICAL INVESTIGATION OF MATERIAL CONSTITUTIVE DYNAMICS}

\section{Problem Statement}

A significant challenge in practical structural engineering is the realistic determination of the parameters that influence the constitutive model of building components. It generally consists of a set of experimental investigations such as stress and bends testing. A finite volume approach for the related mechanical issue creates a direct relationship amongst uncertain variables and observations, which are often local stress elements and tension force in such instances. This method, known as "direct inversion," is briefly discussed below. Nevertheless, such a traditional method has two major flaws that can be stated as follows:

The assumptions required to directly relate unknown factors to pressure and stresses are typically rather strict (for example, uniform stress dispersion at the sample end for a basic tensile strength). For material properties, for example, these criteria are extremely challenging to satisfy. It's much more important when testing diverse components (solder joints, simply supported composites, etc.). Once the constitutive relations depend on more factors than those for isotropy, such experimental investigations can only identify a minimal set of parameters, necessitating the use of multiple analyses (anisotropic elasticity, plasticity for instance).

This remark has prompted numerous writers to propose alternate techniques based on the assessment of diverse stress levels that cannot be reversed immediately (as in mechanical characterization, also known as nonlinear static tests). Both constraints can be circumvented in this situation. Therefore, if the test is properly constructed, the yield stress produces a diverse stress region that includes the entire range of fundamental characteristics. Their continuous recognition therefore is achievable if the reaction is obtained on a sufficient number of data points using an appropriate non - contact assessment technology and a strong classification approach deriving the components from this sort of measured data is available. Entire assessment methods like high - speed photography, speckled and murray detection and ranging, and the array approach have lately gained popularity in the practical mechanical field, prompting some users to question if they can be used for diagnosis.

Because there is usually no closed-form solution providing a direct connection between measures and variable - length, the provision of identification techniques permitting the derivation of constitutive parameters is a significant problem in such approaches. In recent years, several methods have been suggested, including model update approaches and the simulated domains methodology. The basic characteristics of these methods will be presented in this part, and Section III demonstrated their applicability by reviewing some recent instances of their use. One of the goals is to pique the attention of the dynamics of material field, which is increasingly realizing the possibilities of an attempt to handle challenging mechanical characterization problems.

\section{Resolution Techniques}

Direct Inversion

Experimenters have long attempted to create adequate attachments that offer the oxidative stress needed to describe specific resources. The shear modulus is recognized at specified places all over the tested material if this aim is assumed to be already achieved. It is feasible to display the structure's residual stress graphs and infer the governing model that relates the pressure and stress indices directly by collecting the pressure indices around the same places and making some additional hypotheses. As a result, an IP is not required. Eq. (1) has a sigmoid transfer purpose that is clearly understood. To identify an unknown statutory instrument, it is easily reversed. Immediate flip is the name for this method.

Test methods are frequently adequate for technical characterization of unidirectional composites, and they are perfectly suited to the use of immediate reversal. Considering linking factors in the governing equations, this is much more challenging for physical properties, particularly those that vary with respect and nonlinear elasticity. Some resources on the experiments that may be performed to characterize the tensile characteristics of the materials via vertical flip are readily available. As a result, such evaluations may not be created at this location. Basic pressure experiments, inhibition studies, three-point flexural analysis, four-point flexural assessments, transverse direction fatigue test, strain - controlled experiments, Iosipescu compressive test results, off-axis strain gauge for synthetic structures are among the most frequent.

Straightforward translation necessitates the fulfillment of semi criteria. The presence of elastic recoil in earlier experiments makes the connections amongst observations and essential factors more difficult to understand. Via very few 
expectations, immediate reverse can still be employed in the Augmented dickey beam study, but numerous writers have previously highlighted that certain hypotheses are irrelevant, necessitating the resolving of the unknown variable using model update approaches or even other options.

\section{Model Upgrading Techniques}

When the exact reverse of function $\mathrm{g}$ in $u=g(p)$ is unknown, finite element model updating is perhaps the most popular method for identifying variables, driving the Finite Element Model Updating (FEMU). The basic idea behind this method is to create a finite element analysis of the tensile testing under investigation utilizing information on shape and initial conditions. This approach allows for the systematic computation of a variational version of the reaction responding to a variety of parameters, which is indicated $u=g(p)$ in Eq (1). The investigation's identified reaction, indicated as u in $u=$ $g(p)$, could be motions, stress, pressures, and so on. Let $\mathrm{p}$ stand for the collection of material properties to remove, and $0 \mathrm{p}$ stand for the starting estimation of $\mathrm{p}$ required to begin the optimization technique. The gap between $\mathrm{u}$ and $u=g(p)$ is then used to create an objective function. Iterative manners seek routes for reducing the cost variable commencing at $0 \mathrm{p}$ until the preset consolidation threshold is met.

Although the weighted slightest style is popular, it is still not required. Previous knowledge about $\mathrm{u}$, for example, can be conveyed via statistical methodology such as Markov methods or basically by including a punishment element in the optimization problem. Relatively advanced reduction techniques relying on parameter estimation concepts, like the inaccuracy in functionalization, have indeed been applied with effectiveness on rare occasions. A broad range of circumstances can sometimes be explored based on which quantitative variable is utilized for identifying reasons. In reality, it is preferable, but not required, that values obtained be domain variables, since the FEMU approach could be used to almost any type of well over statistics in concept. In reality, an appropriate scheme may be conducted on a collection of data obtained solely on the specimen's border without more assumptions. However, it should be noted that variational structure can be built using numerous mathematical approaches like the partial differential equation, discrete phase approach, or, in some cases, confined approaches.

A significant number of instances of the use of FEMU include experimental findings consisting of comprehensive information can be collected in the research. Identifying flexion tensions in thin samples has been the subject of several investigations [9]. Finite volume responses were used by certain writers to build sample design methodology. As a result, they were able to demonstrate sheet folding trial setups in which the sheet behavior was impacted by all of the rigidity factors that needed to be determined. Similar bend tests were carried out in the lab using substantial timber boards. There is also some fascinating research on the mechanical characteristics of biological materials. The DIC structures situated at the border of the measuring region (close to the cuffs) give the parameter estimation photographer's initial conditions.

Finally, given the broad array of applications, FEMU may be considered a very versatile method. The primary disadvantages are linked to converging difficulties and, as a result, the duration necessary to achieve a resolution ( 23 hours in a previous column for only just a few variables). To resolve such difficulties, a wide range of factors still need to be enhanced: Proper selection of the test case is a critical problem in which significant effort must be dedicated. The majorities of cases, the scientists' experiments are either taken from standard diagnostic settings or developed utilizing sensible guidelines. Except for a few case reports, no comprehensive system testing has indeed been attempted to assure the technology's high specificity to complicating variables, the scientific input feature selection: the FEMU process is evidently hyper delicate to the scientific incoming data selection [10].

The resolution of the Virtual Fields Method (VFM) in comparison to the tension variations is particularly important when addressing heterogeneity tension environments. Furthermore, it is unclear whether information should be used: deformations, stresses, and/or forces, as well as across what portion of the sample. The functional form appears to play a significant impact in the technology's effectiveness. In respect of time complexity and durability, the proposed method selected the responsiveness to misspecification: the objective is to distinguish both identifying mistakes caused by measurement errors and design flaws (boundary conditions, constitutive equations).

\section{CONCLUSION}

The Virtual Fields Method (VFM) seems to be less versatile than Finite Element Model Updating (FEMU) techniques since it requires full-field data, which is a restriction. In reality, complete data is never available in actuality. Although optic and discontinuous techniques may offer dense data over a particular surface or volume, field "u" should always be rebuilt after the VFM is applied, since this field is needed. Even while field u can always be rebuilt in reality from nondense imbalanced datasets, resilience problems during the detection phase may emerge if the pixel size of the observations is not adequate. For issues of elastic moduli determination, the VFM's resilience has been extensively studied. Whenever static noise is introduced to the data, the variance of the variables detected with the VFM is demonstrated to be dependent on the selection of a meaningful collection of simulated fields.

There are optimal simulated fields, which reduce uncertainty and provide the "maximum probability answer." It has also been shown that when field $\mathrm{u}$ is accessible and issues of elastic recognition exist, FEMU methods provide equations that are comparable to those obtained from the VFM, albeit with non-optimal configurations of simulated fields. As a consequence, the FEMU techniques do not provide the "best possible answer." On the other hand, the unpredictability of FEMU techniques varies dramatically dependent on the optimization technique to be reduced. On the one hand, the FEMU technique, which is founded on least-squares 'displacement gap' reduction, generates equations that are very close to that of the VFM technique, and thus its uncertainties is almost equal to the VFM approach. Other methods based on the "normative equation disparity" reduction or the "equilibria loophole" reduction, on the other hand, are proven to yield biased answers. The research comparing the effectiveness of FEMU and VFM formerly stated is solely concerned with the 
detection of elastic moduli. Time complexity seems to be considerably more important in the case of nonlinear elastic or visco-elasto-plastic characteristics. In the FEMU solution to resolve such non-linear inverse problems, the forward problems must be performed a large number of times, which is very costly. The VFM, on the other hand, has shown to be both quick and reliable. As a result, the assumption is that the VFM is the best method for identifying elastic - plastic or poroelastic characteristics from test dataset.

\section{References}

[1]. E. Lee, C. Feigley, J. Hussey and J. Slaven, "Estimation of required monitoring time for obtaining validation data in enclosed spaces", Journal of Environmental Monitoring, vol. 10, no. 11, p. 1350, 2008. Doi : 10.1039/b806421k

[2]. Influenza $\mathrm{A}(\mathrm{H} 1 \mathrm{N1}) \mathrm{v}$ investigation teams, "Modified surveillance of influenza $\mathrm{A}(\mathrm{H} 1 \mathrm{N1}) \mathrm{v}$ virus infections in France", Eurosurveillance, vol. 14, no. 29, 2009. Doi : 10.2807/ese.14.29.19276-en.

[3]. A. R and H. A, "Hierarchical cost effective leach for heterogeneous wireless sensor networks," 2015 International Conference on Advanced Computing and Communication Systems, Jan. 2015.

[4]. K. Frick and O. Scherzer, "Regularization of ill-posed linear equations by the non- stationary augmented Lagrangian method", Journal of Integral Equations and Applications, vol. 22, no. 2, 2010. Doi : 10.1216/jie-2010-22-2-217.

[5]. J. Gunning and M. Sams, "Joint facies and rock properties Bayesian amplitude-versus-offset inversion using Markov random fields", Geophysical Prospecting, vol. 66, no. 5, pp. 904-919, 2018. Doi : 10.1111/1365-2478.12625.

[6]. J. Zhang, "Expectation-maximization algorithms for image processing using multiscale models and mean- field theory, with applications to laser radar range profiling and segmentation", Optical Engineering, vol. 40, no. 7, p. 1287, 2001. Doi : 10.1117/1.1385168.

[7]. S. D and H. A, "AODV Route Discovery and Route Maintenance in MANETs," 2019 5th International Conference on Advanced Computing \& Communication Systems (ICACCS), Mar. 2019

[8]. K. You-Ten, D. Wong, X. Ye, C. Arzola, A. Zand and N. Siddiqui, "Practice of Ultrasound-Guided Palpation of Neck Landmarks Improves Accuracy of External Palpation of the Cricothyroid Membrane", Anesthesia \& Analgesia, vol. 127, no. 6, pp. 1377-1382, 2018. Doi : 10.1213/ane.0000000000003604.

[9]. F. Mathieu, H. Leclerc, F. Hild and S. Roux, "Estimation of Elastoplastic Parameters via Weighted FEMU and Integrated-DIC", Experimental Mechanics, vol. 55, no. 1, pp. 105-119, 2014. Doi : 10.1007/s11340-014-9888-9.

[10]. L. Petureau, P. Doumalin and F. Bremand, "Identification of Local Elastic Parameters in Heterogeneous Materials Using a Parallelized Femu Method", International Journal of Applied Mechanics and Engineering, vol. 24, no. 4, pp. 140-156, 2019. Doi : 10.2478/ijame-2019-0054. 